\title{
Antimicrobial consumption among hospitalized patients with COVID-19 in Pakistan
}

\author{
Zia UI Mustafa ${ }^{1} \cdot$ Muhammad Salman $^{2} \cdot$ Mamoon Aldeyab $^{3} \cdot$ Chia Siang Kow ${ }^{4,5} \cdot$ Syed Shahzad Hasan ${ }^{3,6}$
}

Accepted: 18 May 2021 / Published online: 28 May 2021

(C) The Author(s), under exclusive licence to Springer Nature Switzerland AG 2021

\begin{abstract}
The discovery of different antimicrobial agents has revolutionized the treatment against a variety of infections for many decades, but the emergence of antimicrobial resistance require rigorous measures, even amid the coronavirus disease 2019 (COVID-19) pandemic. This retrospective study aimed to examine the consumption of antibiotics in patients with COVID-19 admitted into the five hospitals in the province of Punjab, Pakistan. We collected data on the consumption of antibiotics, classified using the World Health Organization (WHO) AWaRe (Access, Watch, and Reserve), within two months - August and September, 2020, and the corresponding months in 2019. Consumption of antibiotics was presented as daily define dose (DDD) per 100 occupied bed-days. Eight different classes of antibiotics were prescribed to patients with COVID-19 without culture tests being performed, with the prescribing of antibiotics of the Watch category was especially prevalent. The consumption of antibiotics was higher during the COVID-19 pandemic compared to the pre-pandemic period: the consumption of azithromycin increased from 11.5 DDDs per 100 occupied bed-days in 2019 to 17.0 DDDs per 100 occupied bed-days in 2020, while the consumption of ceftriaxone increased from 20.2 DDDs per 100 occupied bed-days in 2019 to 25.1 DDDs per 100 occupied bed-days in 2020. The current study revealed non-evidence-based utilization of antibiotics among patients with COVID-19 admitted into the hospitals in Pakistan. Evidently, the current COVID-19 pandemic is a public health threat of notable dimensions which has compromised the ongoing antimicrobial stewardship program, potentially leading to the emergence of antimicrobial resistance among pathogens.
\end{abstract}

Keywords Antibiotics · COVID-19 $\cdot$ Hospital $\cdot$ Azithromycin $\cdot$ Resistance

This article is part of the Topical Collection on Covid-19

Chia Siang Kow

chiasiang_93@hotmail.com

1 Department of Pharmacy Services, District Headquarter (DHQ) Hospital, Pakpattan, Pakistan

2 Department of Pharmacy Practice, The University of Lahore, Lahore, Pakistan

3 Department of Pharmacy, University of Huddersfield, Huddersfield, UK

4 School of Postgraduate Studies, International Medical University, Kuala Lumpur, Malaysia

5 School of Pharmacy, Monash University Malaysia, Bandar Sunway, Petaling Jaya, Selangor, Malaysia

6 School of Biomedical Sciences \& Pharmacy, University of Newcastle, Callaghan, Australia

\section{Introduction}

The coronavirus disease 2019 (COVID-19) pandemic has affected more than 220 countries, with over 167 million confirmed cases and 3.4 million deaths worldwide as of May 27, 2021 [1]. In Pakistan, 908,576 laboratory-confirmed COVID19 cases with 20,465 deaths have been reported due to this deadly disease up to the aforementioned date [1]. Around $80 \%$ of patients with COVID-19 manifested asymptomatic or mildto-moderate course of illness while the remaining $20 \%$ developed severe illness [2]. There have been several therapeutic options trialed against COVID-19, but thus far, only systemic glucocorticoids such as dexamethasone are conclusively proven to reduce the risk of COVID-19-related mortality [3]. Particularly, the macrolide antibiotic, azithromycin, with or without antimalarials, had been repurposed as one of the therapeutic options against COVID-19, but the data on the utilization of antimicrobials as a whole in patients with COVID-19 had been scarce throughout the world [4]. 
Despite well-defined instructions from the World Health Organization (WHO) who had called on to reserve the utilization of antibiotics in hospitalized patients with COVID-19 and bacterial co-infection, there had been unjustifiable use in patients with COVID-19, especially among those with a mildto-moderate course of illness without manifesting signs or symptoms of bacterial co-infection [5]. Antibiotics could provide therapeutic benefits in patients with COVID-19 diagnosed with bacterial co-infections, patients who are immunocompromised, or patients with prolonged hospitalization due to their susceptibility to bacterial co-infection [5]. Nevertheless, the signs and symptoms of sepsis from bacterial infection and from severe course of COVID-19 can be challenging to differentiate, and therefore, patients with COVID19 frequently encountered empirical administration of antibiotics. One of the earliest studies from Wuhan, China, revealed that more than $90 \%$ of fatal cases were administered antibiotics without the performance of culture tests [6]. Findings from another rapid review reported that $72 \%$ of patients with COVID-19 were prescribed with antibiotics without rationalizing the need among these individuals [7].

Many low- and middle-income countries including Pakistan are struggling with executing their robust antimicrobial stewardship program (AMS), which had been further deteriorated during this ongoing pandemic. There had been no study conducted in the country evaluating the consumption of antibiotics among hospitalized patients with COVID-19 at the time of writing. Therefore, the current study was undertaken to examine the consumption of antibiotics in patients with COVID-19 admitted into the five hospitals in the province of Punjab, Pakistan.

\section{Materials and Methods}

\section{Study Design and Setting}

This retrospective cohort study was performed in the five hospitals located in the province of Punjab, Pakistan, namely District Headquarter (DHQ) Pakpattan Hospital, Tehsil Headquarter (THQ) Arifwala Hospital, THQ Haveli Lakha Hospital, DHQ Okara South Hospital, and THQ Chichawatni Hospital. The aforementioned hospitals had been allocated with necessary resources for the management of patients with COVID-19 under the administrative control of the Primary \& Secondary Health Care Department, Government of Punjab. Ethical approval of the study was obtained from the Research Ethics Committee, Department of Pharmacy, University of Lahore, Pakistan (REC/DPP/FOP/28). Provisional approval for extraction of data from the electronic health record was obtained from each of the participating hospital.

\section{Study Period and Population}

To assess the potential impact of the COVID-19 pandemic on the consumption of antibiotics in the participating hospitals, the study period was separated into a COVID-19 pandemic period (August 1, 2020 to September 30, 2020), and a corresponding pre-COVID-19 pandemic period in 2019 (August 1, 2019 to September 30, 2019).

We included all consecutive hospitalized patients with the diagnosis of COVID-19 confirmed with real-time reverse transcriptase-polymerase chain reaction (RT-PCR) assay from nasal, oropharyngeal, or nasopharyngeal swab specimens between August 1, 2020 to September 30, 2020. Patients did not meet inclusion criteria if they were only treated in the emergency department without subsequent admission. Patients were also excluded if the diagnosis of COVID-19 was confirmed solely based on signs and symptoms.

Patients with COVID-19 were classified as having mild, moderate, and severe illness. Patients with severe illness were those with one or combination of the following conditions: requirement of mechanical ventilation, requirement of intensive care, respiratory rate $\geq 30$ breaths per minute, and oxygen saturation $\leq 90 \%$. Mild and moderate cases were decided by the treating physician based on the number and/or severity of signs and symptoms.

Patients were identified from the electronic health record of the participating hospitals based on admission into the isolation wards reserved for confirmed or suspected patients with COVID-19. Patients' characteristics including age, gender, comorbidities, severity of illness, blood, sputum, urine, or tissue culture results, medications received during hospitalization, admission into intensive care unit, and death events were extracted anonymously from the electronic health record with a standardized data collection form. Patterns of antibiotic use including indication, dose, duration, frequency, and route of administration were also extracted.

\section{Consumption of Antibiotics and Statistical Analysis}

Consumption of antibiotics was evaluated through weekly measurements of the prescription of antibiotics for systemic use (Anatomical Therapeutic Chemical [ATC] group J01 and azithromycin [ATC J01FA10]). The antibiotics prescribed upon discharge were excluded from this calculation. We used the WHO's AWaRe (Access, Watch, and Reserve) classification to categorize antibiotics for their optimal use. Consumption of antibiotics was calculated as defined daily doses (DDD) per 100 occupied bed-days, in accordance with the ATC and DDD methodology endorsed by the WHO.

Descriptive statistics were used to describe the variables: categorical variables were presented as frequency distribution and percentages, while continuous variables were presented as means \pm standard deviations (SD). 
Table 1 Characteristics of included patients $(\mathrm{n}=$ 202)

\section{Results}

A total of 202 patients (40.1\% were women) with COVID-19 had been admitted to the five district hospitals of Punjab province in August and September 2020, with a mean age of 35.5 $( \pm 10.4)$ (Table 1$)$. The majority $(87.6 \%)$ of patients had mildto-moderate course of illness, with only one death reported within the two months. The most commonly prescribed repurposed drugs for the treatment of COVID-19 were ivermectin $(26.7 \% ; \mathrm{n}=54)$, hydroxychloroquine $(26.2 \% ; \mathrm{n}=53)$, and oseltamivir $(4.5 \% ; \mathrm{n}=9)$.

Antibiotics were prescribed in $88.1 \%$ of the included patients despite no culture tests were performed in these patients to establish bacterial co-infection (Table 1). Eight different classes of antibiotics were prescribed to the included patients with COVID-19. Patients with severe course $(n=25)$ were prescribed with the highest mean number of antibiotics $(2.8 \pm$ $0.8)$, followed by those with moderate course $(1.9 \pm 0.6 ; \mathrm{n}=$ $68)$, and mild course $(1.1 \pm 0.8 ; \mathrm{n}=109)$. The most frequently prescribed antibiotics were azithromycin $(86.6 \% ; \mathrm{n}=175)$, piperacillin-tazobactam $(39.1 \% ; \mathrm{n}=79)$, ceftriaxone $(13.4 \%$; $\mathrm{n}=27)$, ciprofloxacin $(10.9 \% ; \mathrm{n}=22)$, amoxicillin-clavulanic acid $(2.5 \% ; n=5)$, and meropenem $(2.5 \% ; n=5)$. The prescribing of antibiotics in the Watch category was prevalent among hospitalized patients with COVID-19; except for amoxicillin-clavulanate (Access category), the other most commonly prescribed antibiotics are in the Watch category.

Table 2 shows the consumption of antibiotics among the included patients with COVID-19 in the five participating hospitals between August and September, 2020, and the consumption of antibiotics in the corresponding period in 2019 (among non-COVID-19 patients). The consumption of antibiotics was higher during the COVID-19 pandemic compared to the pre-pandemic period: the consumption of azithromycin increased from 11.5 DDDs per 100 occupied bed-days to 17.0 DDDs per 100 occupied bed-days, ceftriaxone from 20.2 DDDs per 100 occupied bed-days to 25.1 DDDs per 100 occupied bed-days, piperacillin-tazobactam from 0.3 DDDs per 100 occupied bed-days to 1.1 DDDs per 100 occupied bed-days, amoxicillin-clavulanic acid from 2.4 DDDs per 100 occupied bed-days to 5.1 DDDs per 100 occupied beddays, and ciprofloxacin from 3.5 DDDs per 100 occupied beddays to 3.8 DDDs per 100 occupied bed-days.

\section{Discussion}

The COVID-19 pandemic has become the greatest global health threat and therefore health care systems around the globe require indispensable allocation of resources for its long-lasting management. It is of immense importance for all the health care providers that concurrent global health

Table 2 Antimicrobial consumption by WHO AWaRe category, for five district hospitals in the province of Punjab, Pakistan

\begin{tabular}{|c|c|c|c|c|}
\hline Study period & Antibiotics & $\begin{array}{l}\text { ATC } \\
\text { code }\end{array}$ & $\begin{array}{l}\text { WHO AWaRe } \\
\text { category }\end{array}$ & $\begin{array}{l}\text { DDDs/ } 100 \\
\text { occupied bed-days }\end{array}$ \\
\hline \multirow[t]{5}{*}{ August-September 2019 (Pre-COVID-19 pandemic) } & Azithromycin & J01FA10 & WATCH & 11.5 \\
\hline & Ciprofloxacin & J01MA02 & WATCH & 3.5 \\
\hline & Ceftriaxone & J01DD04 & WATCH & 20.2 \\
\hline & Amoxicillin-clavulanic acid & J01CR02 & ACCESS & 2.4 \\
\hline & Piperacillin-tazobactam & J01CR05 & WATCH & 0.3 \\
\hline \multirow[t]{5}{*}{ August-September 2020} & Azithromycin & J01FA10 & WATCH & 17.0 \\
\hline & Ciprofloxacin & J01MA02 & WATCH & 3.8 \\
\hline & Ceftriaxone & J01DD04 & WATCH & 25.1 \\
\hline & Amoxicillin-clavulanic acid & J01CR02 & ACCESS & 5.1 \\
\hline & Piperacillin-tazobactam & J01CR05 & WATCH & 1.1 \\
\hline
\end{tabular}


threats like antimicrobial resistance must not be neglected during the time of COVID-19 outbreaks and AMS should be performed as an integral part of the rapid pandemic response. Careful and rational consumption of antimicrobial agents could be significant in the wake of their reserve for those with confirmed bacterial infections or those with signs and symptoms consistent with bacterial infections. Importantly, the Dutch Working Party on Antibiotic Policy recommends obtaining sputum and blood cultures prior to the initiation of empirical antibiotic therapy in patients with COVID-19 pneumonia who have radiological findings and/or inflammatory markers compatible with bacterial co-infection to confirm the diagnosis [8]. They also emphasize the need for appropriate de-escalation of antibiotic therapy based on the results of culture testing [8].

The current study revealed that commonly prescribed antibiotics in hospitalized patients with COVID-19 in Pakistan were azithromycin, ciprofloxacin, ceftriaxone, amoxicillin-clavulanic acid, and piperacillin-tazobactam. The utilization of azithromycin, which was one of the frequently prescribed antibiotics among hospitalized patients with COVID-19 in Pakistani hospitals as reported from our analysis, demonstrated the highest increment in its consumption (as DDD/100 occupied bed-days) as compared to the corresponding time period in the previous year. This could be due to treating physicians' experience of using azithromycin for respiratory infections or they could be influenced by widespread off-label use of azithromycin for the treatment of COVID-19 in other countries. For example, similar findings were reported by a previous study conducted in Singapore, where similar trends of increased azithromycin consumption were reported [9]. This is concerning because acquired macrolide resistance has been an increasingly recognized problem even before the COVID-19 pandemic [10].

A slight increase in the consumption of ciprofloxacin, a fluoroquinolone, was documented in our study. Fluoroquinolones are generally prescribed as an empirical therapy due to their broad-spectrum activity, and typically not for the targeted antimicrobial therapy against causative microorganisms, in order to avoid emergence of antimicrobial resistance. The findings are similar to the previous study conducted by Gonzalez-Zorn in Spain who reported the increased consumption of levofloxacin, another fluoroquinolone, during the COVID-19 pandemic, compared to the pre-pandemic period [11]. Ceftriaxone and piperacillintazobactam are typically prescribed for conditions such as community-acquired pneumonia, hospital- and ventilatoracquired pneumonia. Their utilization also scaled up in Pakistani hospitals after COVID-19 outbreak as compared to the previous year. Our findings are in parallel with that of a study conducted in the United States showing a similar kind of increment in the consumption of ceftriaxone and piperacillin/ tazobactam (as DDD per 100 occupied bed-days) compared with the average consumption before the COVID-19 outbreaks [12]. In fact, the prevalent use of ceftriaxone, a thirdgeneration cephalosporin, should be cautioned due to its association with increased incidence of extended-spectrum- $\beta$ lactamase (ESBL)-producing organisms [13]. A recent study though reported an overall reduction in the incidence of total multidrug-resistant (MDR) bacterial during the COVID-19 pandemic in an Italian university hospital, but there was a significantly higher incidence of MDR bacterial infections in the COVID-19 departments compared with other medical departments (29\% versus 19\%), with ESBL-producing Klebsiella pneumoniae accounted for most of the increments [14].

The current pandemic is a public health threat of notable dimensions which has also compromised the ongoing AMS program, potentially leading to the emergence of antimicrobial resistance among pathogens. Antimircobial resistance has likely been a neglected issue in Pakistani hospitals during the ongoing pandemic that requires prompt attention in order for the effective re-implementation of AMS program. We observed that standard treatment guidelines had been overruled and with increased non-evidence-based antimicrobial consumption among patients with COVID-19. Indeed, antimicrobial resistance should still be recognized as a global health issue that may compromise all the efforts under the umbrella of universal health coverage and the healthrelated sustainable development goal, even amid the COVID-19 pandemic [5]. However, several limitations could affect the generalizability of our findings, which include a retrospective study design and a short study period.

\section{Conclusion}

The current study revealed non-evidence-based antimicrobials consumption among patients with COVID-19 admitted into Pakistani hospitals. Antibiotics were being prescribed without prior establishment of bacterial co-infections where no culture test was performed in these patients. Our findings demand prompt attention in order for the effective re-implementation of AMS program.

Authors' Contributions ZUM and MS contributed equally to the study by writing the first draft and editing further versions of the manuscript. The conceptual idea was developed by ZUM, MS, MA, CSK, and SSH. ZUM, MS, CSK, and SSH collected and analyzed data. All authors critically reviewed the manuscript. All authors read and approved the final manuscript.

Availability of Data and Material All data generated or analyzed during this study are included in this published article.

Code Availability Not applicable 


\section{Declarations}

Ethics Approval and Consent to Participate Ethical approval of the study was obtained from the Research Ethics Committee, Department of Pharmacy, University of Lahore (REC/DPP/FOP/28).

Consent for Publication All authors approved the manuscript for publication.

Conflict of Interest The authors declare no conflict of interest.

\section{References}

1. Dong E, Du H, Gardner L. An interactive web-based dashboard to track COVID-19 in real time. Lancet Infect Dis. 2020. https:// coronavirus.jhu.edu/map.html (28 April 2021).

2. $\mathrm{Wu} \mathrm{Z}$, McGoogan JM. Characteristics of and important lessons from the coronavirus disease 2019 (COVID-19) outbreak in China: summary of a report of 72314 cases from the Chinese Center for Disease Control and Prevention. JAMA. 2020;323(13): 1239-42.

3. Kupferschmidt K, Cohen J. Race to find COVID-19 treatments accelerates. Science. 2020;367(6485):1412-3.

4. Kow CS, Hasan SS. Azithromycin in patients with COVID-19: Friend or foe?. Clin Microbiol Infect. 2021;27(1):136-7.

5. Getahun H, Smith I, Trivedi K, Paulin S, Balkhy HH. Tackling antimicrobial resistance in the COVID-19 pandemic. Bull World Health Organ. 2020;98(7):442-442A.

6. Du Y, Tu L, Zhu P, et al. Clinical features of 85 fatal cases of COVID-19 from Wuhan. A retrospective observational study. Am J Respir Crit Care Med. 2020;201(11):1372-9.
7. Rawson TM, Moore LSP, Zhu N, Ranganathan N, Skolimowska K, Gilchrist $\mathrm{M}$, et al. Bacterial and fungal coinfection in individuals with coronavirus: a rapid review to support COVID-19 antimicrobial prescribing. Clin Infect Dis. 2020;71(9):2459-68.

8. Sieswerda E, de Boer MGJ, Bonten MMJ, Boersma WG, Jonkers RE, Aleva RM, et al. Recommendations for antibacterial therapy in adults with COVID-19 - an evidence based guideline. Clin Microbiol Infect. 2021;27(1):61-6.

9. Liew Y, Lee WH, Tan L, et al. Antimicrobial stewardship programme: a vital resource for hospitals during the global outbreak of coronavirus disease 2019 (COVID-19). Int J Antimicrob Agents. 2020;56(5):106145.

10. Kow CS, Hasan SS. Use of Azithromycin in COVID-19: A cautionary tale. Clin Drug Investig. 2020;40(10):989-90.

11. Gonzalez-Zorn B. Antibiotic use in the COVID-19 crisis in Spain. Clin Microbiol Infect. 2021;27(4):646-7.

12. Nestler MJ, Godbout E, Lee K, Kim J, Noda AJ, Taylor P, et al. Impact of COVID-19 on pneumonia-focused antibiotic use at an academic medical center. Infect Control Hosp Epidemiol. 2020:13.

13. Urbánek K, Kolár M, Lovecková Y, Strojil J, Santavá L. Influence of third-generation cephalosporin utilization on the occurrence of ESBL-positive Klebsiella pneumoniae strains. J Clin Pharm Ther. 2007;32(4):403-8.

14. Bentivegna E, Luciani M, Arcari L, Santino I, Simmaco M, Martelletti P. Reduction of multidrug-resistant (MDR) bacterial infections during the COVID-19 pandemic: a retrospective study. Int J Environ Res Public Health. 2021;18(3):1003.

Publisher's Note Springer Nature remains neutral with regard to jurisdictional claims in published maps and institutional affiliations. 